$\mathbb{T}$ periodica polytechnica

Transportation Engineering $41 / 1(2013) 2531$

doi: $10.3311 /$ PPtr.7105

http://periodicapolytechnica.org/tr

Creative Commons Attribution (i)

RESEARCH ARTICLE

\section{Mini Actuators for Safety Critical Unmanned Aerial Vehicles Avionics}

\author{
Márk Lukátsi / István Réti / Bálint Vanek / Ádám Bakos / József Bokor / István Gőzse
}

Received 2012-11-06

\begin{abstract}
The present article details the development steps and experimental results obtained during the development of smart actuators used on mini unmanned aerial vehicles (UAV). The research effort is driven by the need of developing onboard health monitoring and diagnostics units for small size UAVs to improve their reliability. In the present all small UAVs use single string avionics systems with no built in redundancy, moreover the servo actuators onboard the airplane are often commercial off the shelf (COTS) hobby components with no reliability figures, limited performance guarantees and one directional communication using analog PWM signals. The development of new servo generation focused on solving the above issues. The proposed servo actuators use the existing mechanical gearboxes and housing of the COTS components, but their power electronics, motor control hardware and software components, sensors are custom designed to fit the needs of a higher demand. The actuators with their controlling microprocessors are capable of establishing two way communication via CAN and FlexRay protocol, suitable for safety critical applications, the actuators. The development challenges and experimental results in a hardware-in-theloop (HIL) simulator are discussed in the paper.
\end{abstract}

\section{Keywords}

Mini / Micro UAV · Smart Actuator · Electromechanical Actuator $\cdot$ Safety Critical Systems $\cdot$ Flexray communication

\section{Márk Lukátsi}

Systems and Control Laboratory, Computer and Automation Research Institute, Hungarian Academy of Sciences, Kende u. 13-17., H-1111 Budapest, Hungary e-mail: lukatsi88@gmail.com

\section{István Réti \\ Bálint Vanek \\ Ádám Bakos \\ József Bokor \\ István Gőzse}

Systems and Control Laboratory, Computer and Automation Research Institute, Hungarian Academy of Sciences, Kende u. 13-17., H-1111 Budapest, Hungary

\section{Unmanned Aerial Vehicles}

The emerging role of Unmanned Aerial Systems (UAS) for both military and civil operations depends on the ability to gain unrestricted access to national airspace [3, 8]. One of the key issues that must be resolved to open up the skies for UAS is to be able to coexist safely and effectively with current manned operations in the national and international airspace. This includes the ability to follow pilot commands with high fidelity even in the case of component faults. Since current UAVs, with the exception of Predator, Global Hawk and a few other high cost systems, use single string avionics, there is no way of mitigating flight control system component faults during flight [2]. It is our aim to develop a redundant low-cost avionics system for UAVs [14, 15], where hardware redundancy is combined with analytical redundancy to reduce the overall weight and cost, but take advantage of increased computational performance onboard the aircraft.

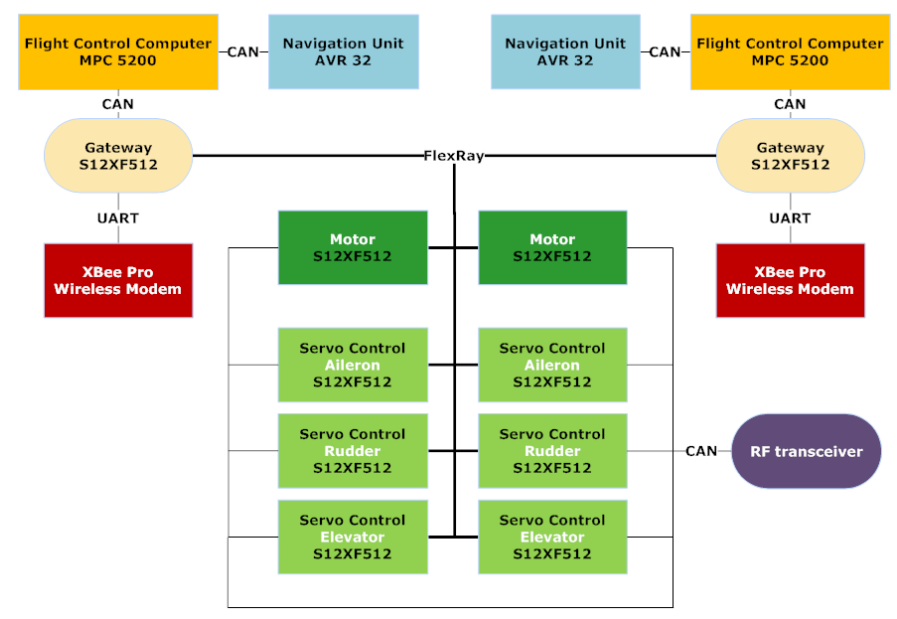

Fig. 1. Interconnection of the UAV avionics

The avionics system is based on the philosophy, that in most situations a carefully selected set of built-in-tests and proper handing over protocols between parallel channels can provide the necessary reliability figures [13]. In case two flight control computers are used and one fails the other will be able to clearly identify the event of a fault in almost all situations if we assume 

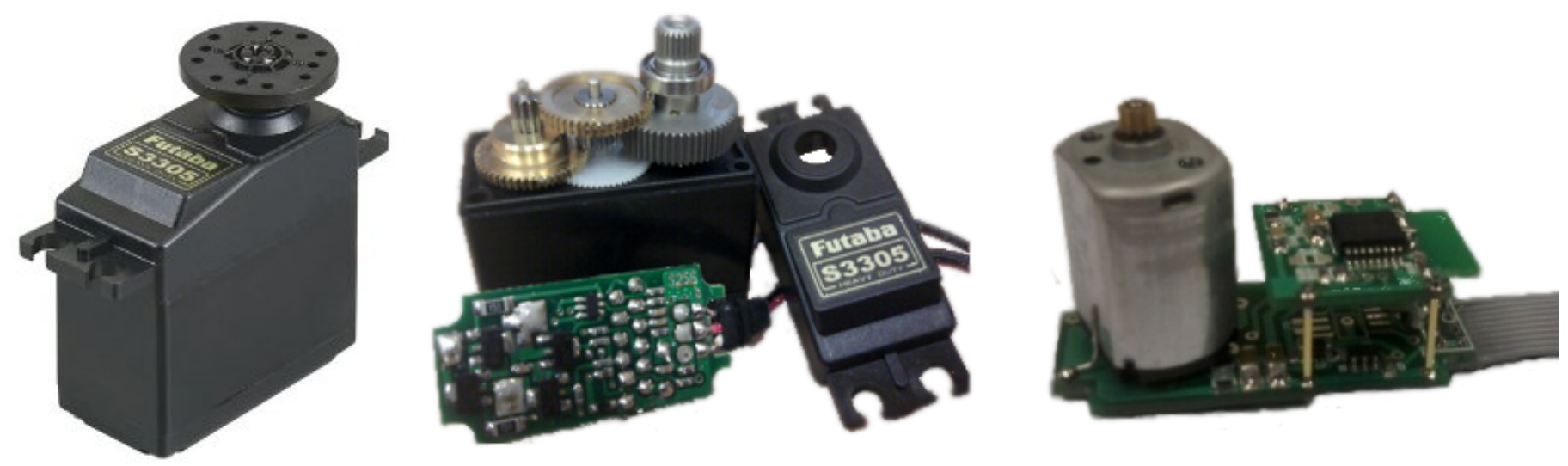

Fig. 2. Futaba S3305 COTS RC servo and the custom servo with measurement and power electronics

the failed node is transmitting random messages not intentionally trying to attack the rest of the system. The system architecture developed in SZTAKI (Computer and Automation Research Institute of the Hungarian Academy of Sciences) can be seen in Fig1 It consists of two independent flight control computers, two INS/GPS sensor units, the three major motion axes are controlled by pairs of independent flight control surfaces, the aircraft has two engines with their own dedicated batteries, two independent electrical power sources are fed to each avionics component and the avionics components are interconnected with a safety critical FlexRay communication bus [9]. The overall architecture, in its simplest form consists of 12 smart units, each having its own computational capability, which allows to transmit two directional messages between Flight Control Computers (FCC) and actuators [15]. In conventional small size UAV applications the FCC only sends analog commands to the actuators and might receive an analog feedback from a position sensor about the current status of the unit. In our approach the FCC sends commands over a digital channel to the actuators, where the smart unit takes care of the internal control tasks of servo control and Pulse Width Modulation (PWM) control of the DC motor inside the actuator. Besides the local control tasks the unit is also capable of providing fault detection capabilities [12], since position, back electromotive force $\rrbracket^{1}$ and drawn current are all measured and using the mathematical model of the actuator analytic parity relations can be used to identify anomalous behavior.

FlexRay communication protocol is selected to provide interconnection between the nodes due to its low cost and the availability of development tools [14]. A consortium including BMW, DaimlerChrysler, Motorola, and Philips, has developed FlexRay for powertrain and chassis control in cars. It differs from conventional buses like CAN or LIN, since its operation is divided between time-triggered and event- triggered activities. Published descriptions of the FlexRay protocols and im-

\footnotetext{
${ }^{1}$ Back EMF is the voltage induced by the motor, when no current is drawn (no voltage is applied), from which RPM can also be measured at high rotation rate.
}

plementation are described in [9]. In both cases, duplication of the interconnect is optional. Each FlexRay interface (it is called a communication controller) drives the lines to its interconnects through separate bus guardians located with the interface. (This means that with two buses, each node has three clocks: one for the controller and one for each of the two guardians; this differs from the bus configuration of TTA, an alternative time-triggered protocol [18], where there is one clock for the controller and both guardians share a second clock.) Like the bus configuration of TTA, the guardians of FlexRay are not fully independent of their controllers.

\section{Servo Actuator}

The UAV under development is based on a hobby RC aircraft frame, which is modified for the research purpose of autonomous flight and development of a vision based sense and avoid system [11]. Since the airframe is based on a hobby aircraft, the servo mounting positions and place for servos is based on commercially available units. Hence, it is practical to develop custom servos with the same form factor as the standard ones available, more over the gearbox, housing and DC motor can be re-used. On the other hand the onboard electronics of a COTS servo is a black box for the user, hence it cannot be modified for research purposes. Moreover, they do not satisfy the requirements of safety critical applications, they are built from a few standard components with minimum "intelligence" in their control logics:

- The control is done with a dedicated printed circuit board, in this form there is no way of modifying its behavior

- Servo shaft angle (motor shaft after the reduction gears) is measured with a potentiometer

- Induced voltage of the motor is measured

- Voltage regulation is done via a MOSFET bridge

- The reference signal is implemented with a $0-5 \mathrm{~V}$ level PWM input, this corresponds to a $50 \mathrm{~Hz}$ frequency square wave signal with different pulse widths. Maximum displacement is 
commanded with $1 \mathrm{~ms}$ long high and $19 \mathrm{~ms}$ long low signal value, while negative sign maximum displacement is achieved with $2 \mathrm{~ms}$ long high signal level.

- The difference between maximum and minimum displacement is less than 270 degrees, limited by the mechanical construction of the potentiometer

- Communication with the environment is one-way, via the ana$\log$ PWM signal.

Due to the aforementioned limitations, COTS servos are not applicable for safety critical UAV applications, the custom made servo has to satisfy the following requirements:

- Independent, self-contained operation with multiple cascade control-loops, reference tracking with sufficient bandwidth and zero steady state error

- The control-loop parameters should be tunable, to achieve different desired responses

- To satisfy the model based control and fault detection requirements, the model parameters should be measured or identified

- All the measurable quantities should be available for diagnostic purposes, to provide the highest number of analytically redundant data

- Self-testing and self-diagnostics should be implemented

- High-level, two-way communication via the FlexRay avionics bus should be implemented

- The lifespan of the servo due to customization should not be compromised

A smart actuator satisfying the performance requirements above can serve as a smart-unit onboard the safety critical UAV.

The first task is to select a suitable servo type for modification. The three main requirements are precision, maximum torque and lifespan. Since only the housing, gears and the motor is used in the modified servo, these requirements pointed towards a unit with metal gears, small backlash and with sufficient space in the housing. The motor should be coreless, since it is free from the reluctance type torque disturbances, which makes the characteristics of the motor magnetic field nonlinear around small torques, undesirable for control purposes. We used a COTS Futaba S3305 RC servo [5] as a baseline, which is modified during the development of the custom actuator unit as shown in Fig. 2. This has a non-coreless motor, but the gears are metal with minimal backlash. In a later stage of development we will consider to replace it by a coreless one.

In the second stage of the development, the electronics modules of the unit are developed. According to the specification, the servo should be able to communicate via the FlexRay bus. Since this communication protocol is not widespread in the industry, due to its maturity, only a limited set of microcontrollers have communication controllers built into them supporting the FlexRay protocol. Our choice was to use the Freescale S12XF512 microcontroller, which has a development environment and available not only for automotive customers. This unit is a relatively large integrated circuit, with 112 legs, which is larger than the size of the servo housing, hence the complete electronics is done in two separate components. The board housing the sensors, power electronics and the control electronics is placed inside the servo unit, while the board containing the S12XF microcontroller is outside the box, connected via a dedicated cable, as shown in Fig. 3. The module containing the microcontroller is designed to be able to control not only the servos but the large BLDC electrical engines of the aircraft via their dedicated power electronics. The units also have a CAN bus communication link as a backup. In case manual override is necessary the pilot is directly controlling servos via a dedicated CAN link between the receiver and the servos.

The electronics inside the servo contains the following components:

- Sensor for the angle measurement of the servo shaft

- Circuit, including signal conditioning and amplification, of the induced voltage measurement in the motor

- Circuit, including signal conditioning and amplification, of the current measurement inside the motor

- The MOSFET bridge, controlling the motor voltages, with its driving circuit

The magnetic sensor to measure shaft position is the Austria Micro Systems AS5045 unit which is a system on a chip solution for Hall-element sensing. The analog amplification and digital signal processing is done on the unit, measurement are sent via an SPI communication bus. It allows contactless angle measurement with 12 bit resolution leading to $0.0875^{\circ}$ maximum precision.

The signals sent to the microcontroller board, outside are the digital busses of measured current, voltage, and the position of the shaft, and the digital signals sent from the microcontroller are the sign of motor rotation and the voltage on the motor. The supply voltage for the motor and for the electronics are also sent via this cable. The control, fault diagnostics and communication algorithms are implemented on the microcontroller, which has access to all internal and the necessary external signals. The circuit board is connected to the redundant electrical network onboard the aircraft, via a power switch, which is selecting always the healthier electrical bus with seamless transition.

It is worth to note, that each unit is also equipped with a CAN communication network, which serves as a direct backup communication link, in case manual flight is required by the safety pilot. In this case the $\mathrm{RC}$ receiver signals are captured from the receiver with a PIC24F microcontroller and sent directly to the 


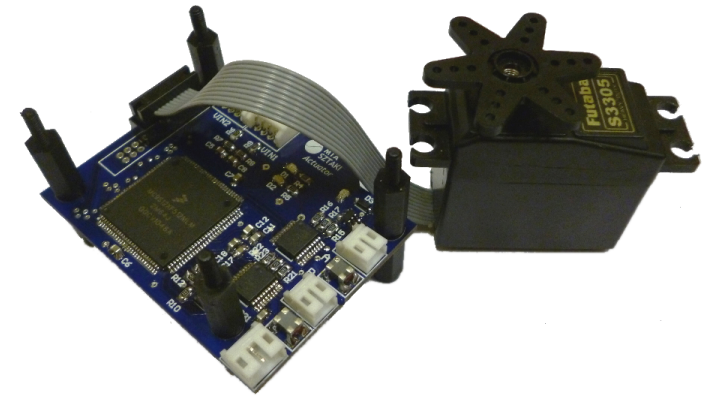

Fig. 3. Modified servo with the external S12XF microcontroller.

servos, bypassing the FCC and the FlexRay communication network.

\section{Implementation}

The main goal of the custom servo development is precise position control. To close the position loop in the controller an additional inner angular rate loop based on back EMF measurement is necessary. It is interesting to note, that this signal can be measured only when there is no voltage applied to the motor (at zero PWM level), after the transients. A dedicated logic is determining the required PWM signal and based on the rotation speed the sequence of polarities applied to the motor to maintain the desired rotation direction, since only rate but not the direction is determined by the PWM signal.

The logic behind the control is the following: position control is done with $250 \mathrm{~Hz}$, with position measurement of $250 \mathrm{~Hz}$. The frequency of the PWM signal is $1 \mathrm{kHz}$, back EMF is measured with this frequency and speed control is also done with $1 \mathrm{kHz}$. It is also important to note, that back EMF is always measured in the middle of the low PWM level, to reduce the transient effects.

The control is done via two cascade loops [19], where the inner loop is realized as a PI controller and the outer loop as a $\mathrm{P}$ controller. The inner loop is responsible for angular rate while the outer loop for angle reference tracking as shown in Fig. 4 (saturation of angular rate and PWM increments are included in the controller blocks). As a first step, the model of the actuator is identified, using subspace based identification methods. PWM steps are used as input signals, while back EMF and shaft angle measurements are used as outputs. The identified model has one dominant and four minor modes, hence a simple first order model is selected for control design purposes:

$$
G_{\text {nom }}=\frac{2.836}{s+7.586}
$$

where the input of the model is PWM increment, which has values between \pm 1750 , while the output is angular rate of the output shaft in $\%$ sec. The identification is done around $0 \% \mathrm{sec}$ angular rate, but it is important to note that a maximum angular rate of $420 \% \mathrm{sec}$ is achievable with the design and at higher angular rates the system becomes strongly nonlinear. The inner loop controller has the form:

$$
C_{i}=A_{i} \frac{1+s T_{I}}{s T_{I}}
$$

The integrator gain $T_{I}$ is selected to have a pole zero cancellation at $T_{I}=1 / 7.586$ and the steady state gain is selected to avoid saturation $\left(A_{i}=10\right)$. In principle the open-loop is now an integrator. The closed loop is augmented with an integrator to provide angle response:

$$
G_{i, c l}=\frac{28.36}{s(s+28.36)}
$$

This model has angular rate reference input in $\% \mathrm{sec}$ and angle as output in ${ }^{\circ}$. Theoretically the outer controller is a PD controller with a zero cancelling the pole of $G_{i, c l}$, but omitting the derivative term gives better results. This effect may be accounted to the nonlinearities present at higher angular rates. The static gain is chosen to avoid saturation $\left(A_{o}=2\right)$.

Careful measurement of the back EMF signal is essential which is proportional to the angular rate in a wide range. Measurement is implemented with the use of the PWM counter interrupt which is triggered when the counter reaches zero. This ensures that the applied voltage is zero on the motor and the measurement takes place in the middle of the stage after the transients faded out. The measurements are still corrupted with noise, hence a low pass filter with $40 \mathrm{~Hz}$ corner frequency is used to smooth the signal. It is also important to note, that the output of the controller is PWM signal, and the maximum allowed PWM command has to be limited to allow enough time for back EMF measurement. After several iterations, the maximum PWM level is limited to $68 \%$, with this the EMF measurement is not compromised. Since the system dynamics have lower gain in higher angular rate range, this does not limit the performance of the servo, but an input limit of $420 \% \mathrm{sec}$, corresponding to the maximum PWM level have to be imposed on the reference commands. The inner loop controller has one additional feature, in case a constant disturbance is acting on the surface the integral of the error grows fast, which leads to large transients after the disturbance is gone. To handle this problem a proper anti-windup strategy is necessary to limit the transients. The difference between the maximum PWM level and the output of the controller multiplied by the anti-windup gain $\left(A_{a w}=1 / 150\right)$ is subtracted from the input of the integrator in case the maximum level is exceeded.

The outer, angle tracking loop is done with $250 \mathrm{~Hz}$ update rate. Angle tracking with large deflections is handled well, but due to the backlash in the gearbox and the high mechanical friction, precise positioning is challenging. With a simple $\mathrm{P}$ control structure the servo output is oscillating around the reference angle with approximately $10 \mathrm{~Hz}$ frequency with $5^{\circ}$ amplitude due to the friction of the gears inside the housing. To better handle the situation an empirical extension is made to increase the controller gain at smaller errors. If the error is smaller than 50 PWM increments the controller gain is increased linearly, reaching $3 A_{o}$ when the error reaches zero. This eliminates the problems related to friction and backlash.

Considering the assembly of the magnetic rotary encoder, due 


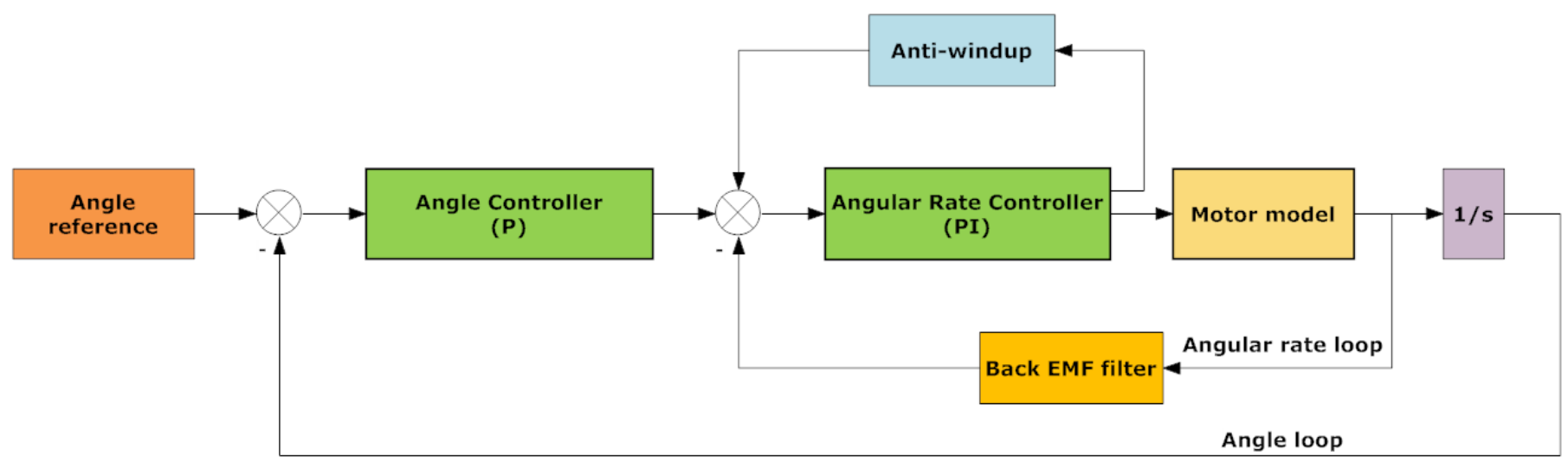

Fig. 4. PID control loop implementation of the actuator servo

to the shift of the shafts a periodic offset error obtains at angle measurement. This cyclic error is induced by the difference of the unwrapped, and detrended shaft angle and its fitted polynom (shown in Fig. 5). After a sufficient number of measurements, within the confidence interval of the periodic errors, the most likely offset values - for a complete turn - are obtained by averaging. Finally quantization is needed, to get sensor increment dimension for the software implementation. After compensation, the control characteristics are only slightly affected, however the absolute position measurement is improved up to a significant value of $2^{\circ}$.

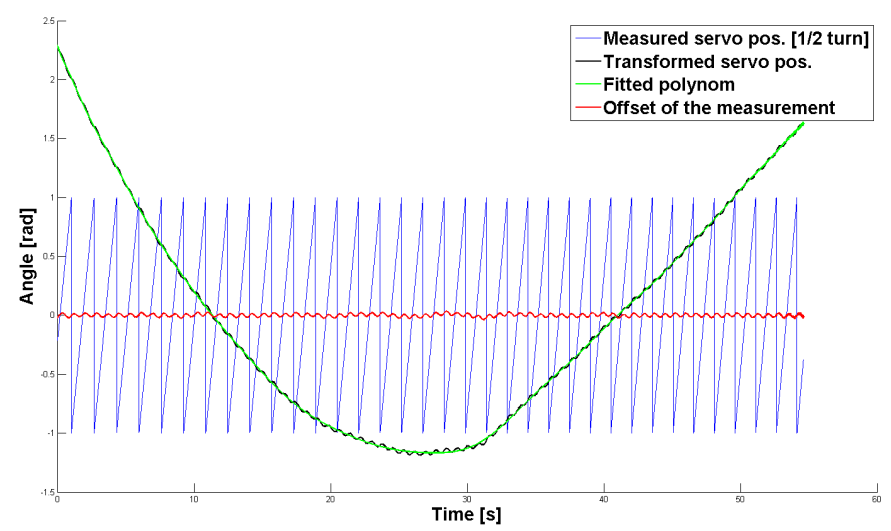

Fig. 5. Magnetic rotary encoder compensation

\section{Test results}

The behaviour of the servo is compared with an off the shelf servo unit (Futaba SG5010) as shown in Fig. 7) The response for a step command of $30^{\circ}$ is compared, where the advantage of the custom made servo is clearly visible. The measurement from the factory servo is corrupted by noise, but it is evident that the settling time and the overshoot are both larger in this case, moreover the factory servo has higher steady state error.

The ultimate goal of the research is to use the actuator onboard the development UAV [1], but before flight testing, the unit has to prove its performance and reliability. A hardware-inthe-loop test environment is used to test the FCC and the implemented control system (shown in Fig. 6). In its original form the PWM signals generated by the main FCC are sent back to the aircraft simulation via a PWM capture card and the actuator dynamics are omitted from the simulation. However, with the current simulation set-up the real actual position of the actuators, along with other measurements useful for health monitoring, are sent back to drive the aircraft dynamics providing a more realistic simulation. The actuators are connected to the FCC via the FlexRay network and the measurements are sent back to the simulation via a serial link, so the PWM capture card is removed from the set-up.

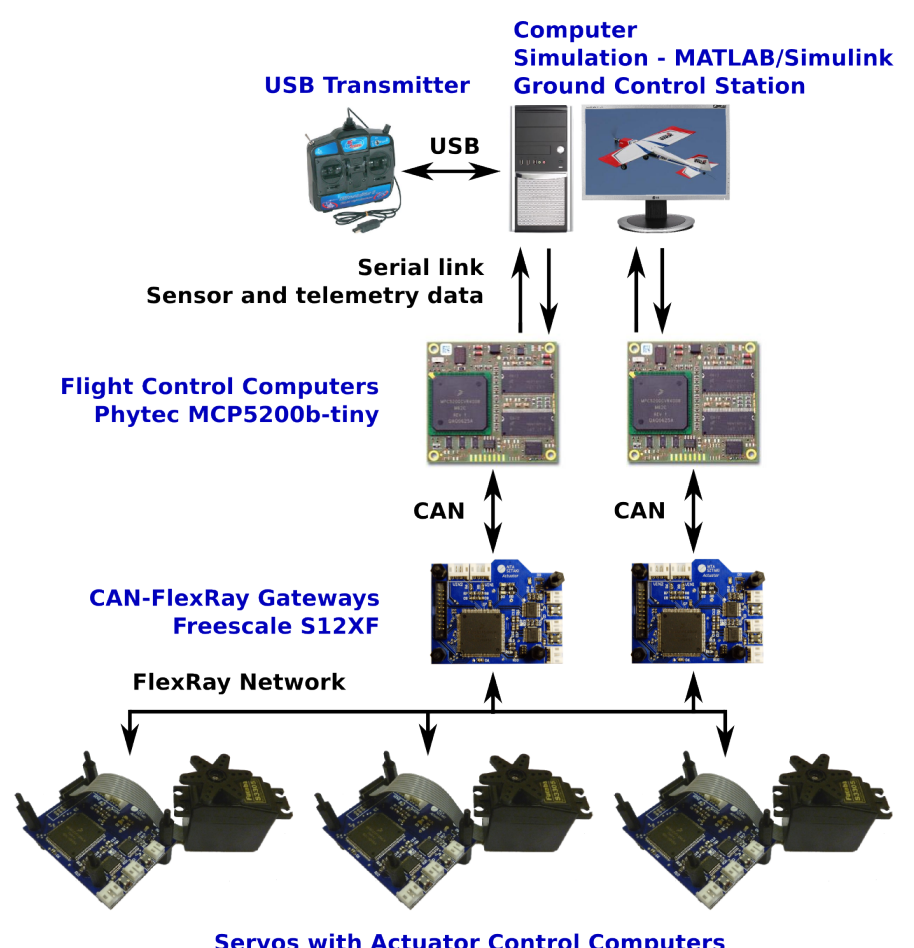

Fig. 6. Hardware-in-the-loop setup for UAV development

Actuator deflections are shown in Fig. 9. It can be seen that the reference signal is tracked well and the steady error is smaller than $0.4^{\circ}$, which is acceptable for flight control.

The servos were tested for 30 hours in continuous waypoint navigation flight, in the first 10 hours with no load, in the second 10 hours with loads corresponding to the aerodynamic forces expected during operation, and then another 10 hours with no load. The tests were accomplished with no software or hard- 
ware faults. The temperature of the servos stabilize at $45 \mathrm{Cel}-$ sius, within 10 minutes after warmup, and the power consumption was also fairly constant in the first 10 hours. It increased after applying the load, and unexpectedly did not go back to the original level after removing the load, which might be caused by the wear and tear during the testing.

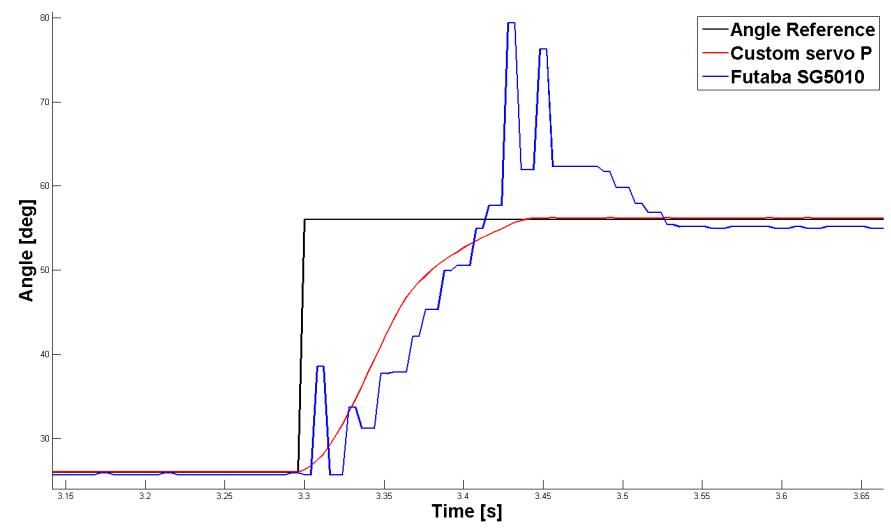

Fig. 7. Comparison of Futaba SG5010 and custom servo

The servo is proven to be reliable enough for flight tests and one of the units is installed to the development UAV. The servo receives the same reference signal as the one corresponding to the rudder control surface, but it lacks physical connection with any of the control surfaces. Deflection is shown in Fig. 8 during a manual flight test.

\section{Conclusion}

The present article discusses the development of a smart actuator used on a small scale UAV. The newly developed servo unit builds heavily on the mechanical components of a COTS RC servo unit, but its electronics an software are custom designed for the purpose of a fault-tolerant safety critical avionics system. The reasons behind design decisions are discussed and the development steps are detailed in the article, followed by experimental results done on a hardware-in-the-loop test facility. The future steps should include the characterization of dominant fault modes of the actuator, along with determining the reliability figures of the units including mean time between failures and evaluation of the performance of the onboard health monitoring unit (true detection rate, missed detection rate, false alarms).

\section{Acknowledgement}

The project has been supported by the Foundation for Hungarian Transportation Engineering and HungaroControl, the Hungarian Air NAVIGATION Services Co.

\section{References}

1 Bauer P, Chai PY, lannelli L, Pandita R, Regula G, Vanek B, Balas GJ, Glielmo L, Bokor J, UAV Lab, open research platform for unmanned aerial vehicles, In: Advances in aerospace guidance, navigation and control. Selected papers of the 1st CEAS specialist conference on guidance, navigation and control; Munich, Germany, 2011, DOI 10.1007/978-3-642-19817-5_14

2 Cox TH, Nagy CJ, Skoog MA, Somers A, Warner R, Civil UAV Capability Assessment, NASA Dryden Flight Research Center, 2004.

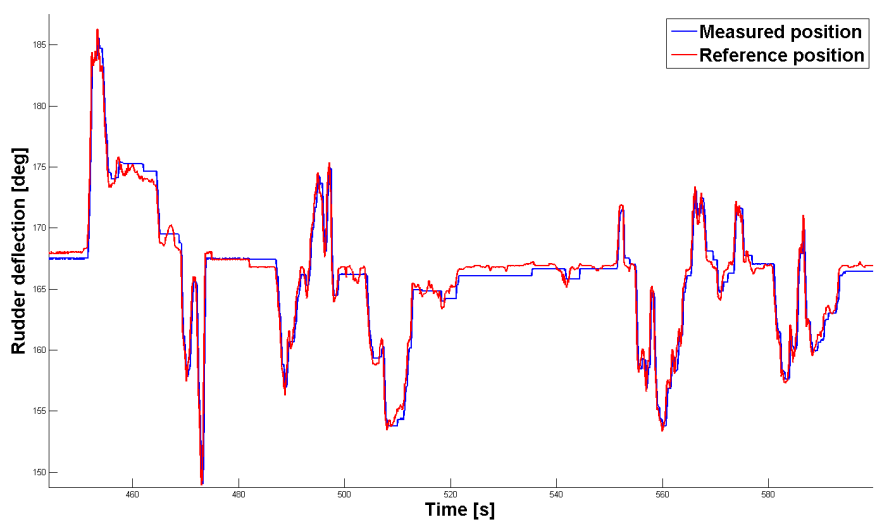

Fig. 8. Rudder deflection during a manual flight test

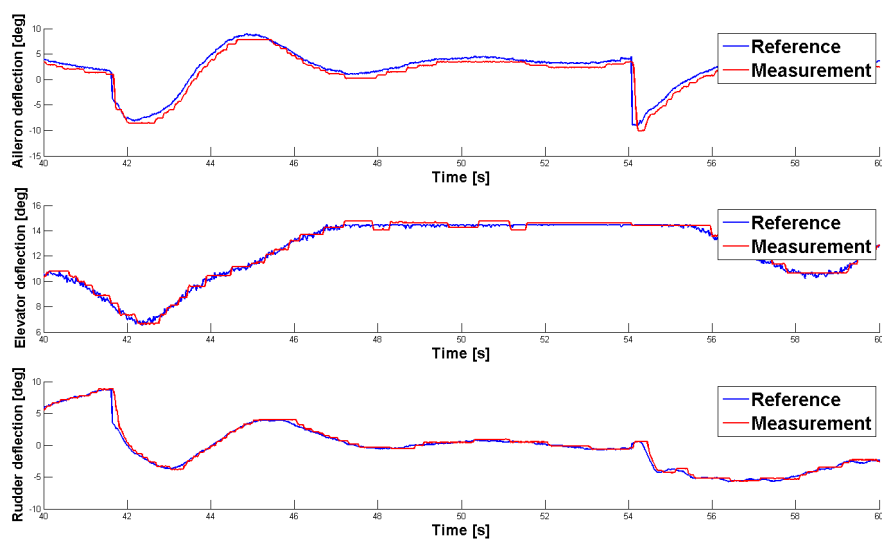

Fig. 9. Actuator deflections in a hardware-in-the-loop simulation

3 Dempsey ME, U.S. Army Unmanned Aircraft Systems Roadmap 2010-2035, U.S. Army UAS Center of Excellence, 2010.

4 FAA, Automatic Dependent Surveillance Broadcast (ADS-B) Out Performance Requirements To Support Air Traffic Control (ATC) Service, Federal Register, 75, (2010), 30160-30194.

5 Futaba Corp., S3305 Servo Manual, (2012).

6 Kurlak RP, Chobot JR, CPU Coverage Evaluation Using Automatic Fault Injection, AIAA Journal on Guidance, 5, (1982), 573-577, DOI 10.2514/3.19791

7 McGough J, Mulcare D, Larsen WE, A method of measuring fault latency in a digital fight control system, In: AIAA/IEEE Digital Avionics Systems Conference, 1988, DOI 10.2514/6.1988-3863

8 United States Government Accountability Office, UNMANNED AIRCRAFT SYSTEMS, Measuring Progress and Addressing Potential Privacy Concerns Would Facilitate Integration into the National Airspace System, 2012.

9 FlexRay Communications System Protocol Specification Version 3.0.1, Adam Opel and Bayerische Motoren Werke, Daimler and Freescale Halbleiter Deutschland, NXP B.V., Robert Bosch, Volkswagen, 2010.

10 Polenz S, Cake F, Görke S, Küke R, Reichel R, SAFAR Eine Fly-by-Wire Steuerung für ein Flugzeug der General Aviation (Diamond DA42), In: 60 Deutscher Luft- und Raumfahrtkongress, 2011.

11 Vanek B, Péni T, Zsedrovits T, Zarándy Á, Bokor J, Roska T, Performance analysis of a vision only Sense and Avoid system for small UAVs., In: AIAA Gudance, Navigation and Control Conference, 2011.

12 Vanek B, Szabó Z, Edelmayer A, Bokor J, Geometric LPV fault detection filter design for commercial aircrafts, In: AIAA guidance, navigation, and control conference; Portland, USA, 2011 
13 Varga T, Architectural Considerations of a Safety Critical UAV Control System, Budapest University of Technology and Economics, 2011.

14 Réti I, Design and Implementation of an UAV Onboard Communication System, Budapest University of Technology and Economics, 2012.

15 Lukátsi M, Avionics Architecture for Safety Critical UAVs, Budapest University of Technology and Economics, 2012.

16 Yeh YC, Triple-triple redundant 777 primary flight computer, In: IEEE Aerospace Applications Conference, 1996.

17 Vanek B, and Szabó Z, Edelmayer A, Bokor J, Robust model matching for geometric fault detection filters: a commercial aircraft example, In: Preprints of the 18th IFAC World Congress, Milano, 2011.

18 Kopetz H, Bauer G, The time-triggered architecture, Proceedings of the IEEE, 91(1), (2003), 112-126, DOI 10.1109/JPROC.2002.805821

19 Zaccarian L, DC motors: dynamic model and control techniques, In: Lecture Notes; Roma, Italy, 2012. 